\title{
THE APPLICATION OF WIRELESS NETWORK DATA ACQUISITION TECHNOLOGY IN HEATING SYSTEM
}

\author{
Fusheng Fan, Wei Liu*, Xuezhong Ai*, Gang Fang \\ Department of control engineering, Jilin Institute of Chemical Technology, No. 45, Chengde street, Longtan District, Jilin, China. \\ *Corresponding Author Email: 13843227008@163.com
}

This is an open access article distributed under the Creative Commons Attribution License, which permits unrestricted use, distribution, and reproduction in any medium, provided the original work is properly cited

\section{ARTICLE DETAILS}

\section{Article History:}

Received 26 June 2018 Accepted 2 July 2018

Available online 1 August 2018

\section{ABSTRACT}

This paper mainly adopts STM32 embedded microprocessor technology, ZigBee short-range wireless ad hoc network communication technology, Internet technology and Internet communications network database technology to achieve dynamic urban heating remote control and management, to achieve energy saving, real-time dynamic control of heating, heating quality monitoring evaluation and so on, in order to reduce heating company and the work intensity of heating management and to improve work efficiency. At the same time, "temperature area method" heat metering program is put forward to provide technical support for the realization of dynamic heating charges. Among these, the paper mainly focuses on the design of building concentrator.

\section{KEYWORDS}

GPRS, Zigbee module, data concentrator, urban heating, dynamic adjustment of heating.

\section{INTRODUCTION}

The According to the traditional heating mode, it is often impossible to guarantee the quality of heating for residents who are far away. However the neighboring resident will get more heat than they need, resulting in waste of resources. In order to guarantee the quality of heating, the method of dynamic adjustment of heating is adopted to optimize the heat distribution problem of "temperature area method". In the whole system, the data aggregation transmission feedback is the most important, considering the actual application needs, the whole system needs to reduce the cost and power consumption. The main building concentrator uses HUAWEI MG323AGPRS wireless communication module, using Zigbee embedded module collocation. Practice has proved that the design of wireless data acquisition and transmission concentrator is feasible, because it has the following features such as low power consumption, high communication speed, stable data transmission, without the need to set up the network, and free of maintenance etc [1].

Through using Internet technology, GPRS mobile Internet technology and short-range ad hoc network Zigbee wireless communication technology, a set of heating system with features of energy saving, control, evaluation and management has been designed and implemented in order to save energy, improve work efficiency. The significances of this project mainly include the following points:

- Low Carbon and Energy-saving: reduce unnecessary heat loss and reduce emissions;

- Dynamic Control: dynamic control of each heat exchanger has been conducted according to the heat supply situation of each neighborhood;

- Heating Monitoring: to provide a basis for the heating company and government heating management to evaluate the quality of heating;

- Dynamic charges: to provide technical support for dynamic heating charging "temperature area method";
- Increase efficiency: improve the efficiency of heat supply and management on the whole.

\section{THE SYSTEMATIC DESIGN}

Building concentrator: Each building has one concentrator. The building concentrator is responsible for making communication between each corridor controller with an Internet server. The data in connection with the indoor temperature collected by the temperature collector transmits to the building concentrator through the ZigBee network, and the data will be uploaded to the server through the GPRS network of the building concentrator. Building concentrator is mainly composed of STM32 main controller, GPRS wireless module, ZigBee low-power ad-Network wireless communication unit and valve and valve control circuit [2].

STM32chip, which has stable and reliable property, has been chosen as master chip with the purpose of meeting the requirements for the transmission rate in complex environments. It mainly possesses the following features such as the high-performance, real-time, micro-power, low-voltage and so on, at the same time, maintaining a high level of integration and ease of development. Huawei MG323A GPRS wireless module use industry standard interface. $\mathrm{mg} 323$ which has the function of gsm/gprs、gsm 850、gsm900、gsm1800a and gsm1900mhz, as a small size and low power consumption, realize the high-speed transmission of voice, SMS, data and fax information. Zigbee module selects SZ05 series of embedded wireless communication module, and the RF transceiver integrated microprocessor Zigbee protocol standards compliance, which has advantages of a communication distance, anti-interference ability, flexible networking, reliable and stable performance [3]. Transparent transmission between devices can be achieved between point to point, one point to multiple points, multipoint to multipoint. This can be composed of star, tree and mesh network honeycomb structure. The main system circuit STM32 mainly has control circuit, a communication circuit, the Mbus circuit, thermal valve table circuit, a memory circuit, a display circuit, a power supply circuit, Zigbee communication circuit and GPRS communication circuit. The system shown in Figure 1: 


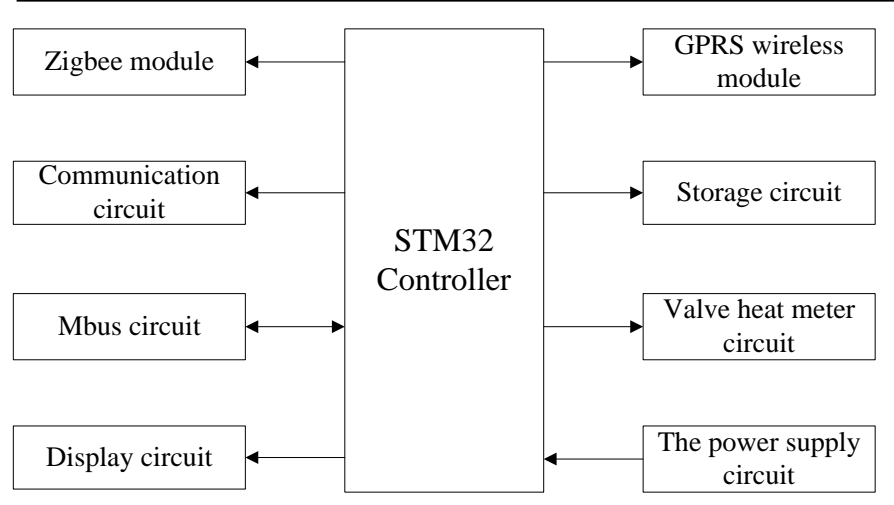

Figure 1: Diagram of the system

\section{CIRCUIT DESIGN AND DATA TRANSMISSION FLOW OF GPRS WIRELESS MODULE AND ZIGBEE MODULE GPRS}

\subsection{The design of GPRS wireless module system}

The system GPRS wireless system mainly consists of STM32 core chip, Mg323agprs module design, power module design and communication module design. Among these, the internal circuit of Mg323agprs Wireless module is mainly composed of mg323a core chip circuit, power supply circuit, SIM card circuit, switching machine reset and LED display circuit and TTL serial port circuit. It also possesses SMS and voice services, with the maximum downlink transmission rate of $85.6 \mathrm{kbps}$, the maximum uplink transmission rate of $42.8 \mathrm{kbps}$, through the AT command to complete remote monitoring. The internal block diagram of MG323A GPRS Wireless module has been shown in Figure 2 [4].

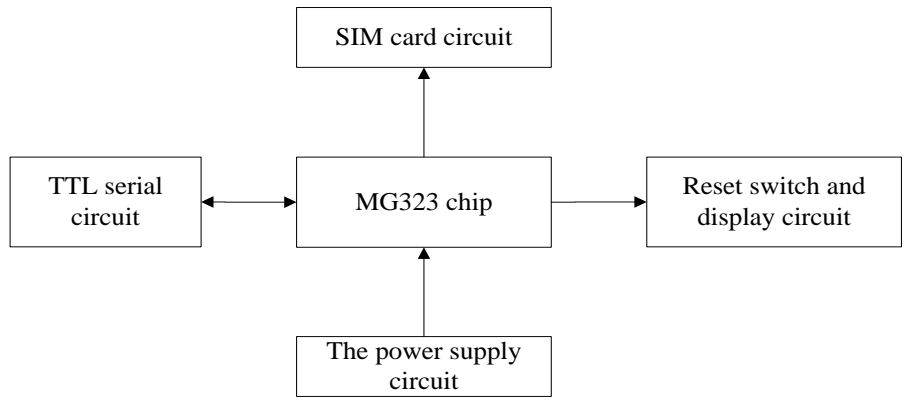

Figure 2: MG323A GPRS wireless module diagram

As a relatively mature module in wireless communication system, MG323 module has the characteristics of simple and convenient circuit connection, wide application range and fast communication speed. The schematic diagram of MG323 interface circuit is shown in Figure 3 [5]

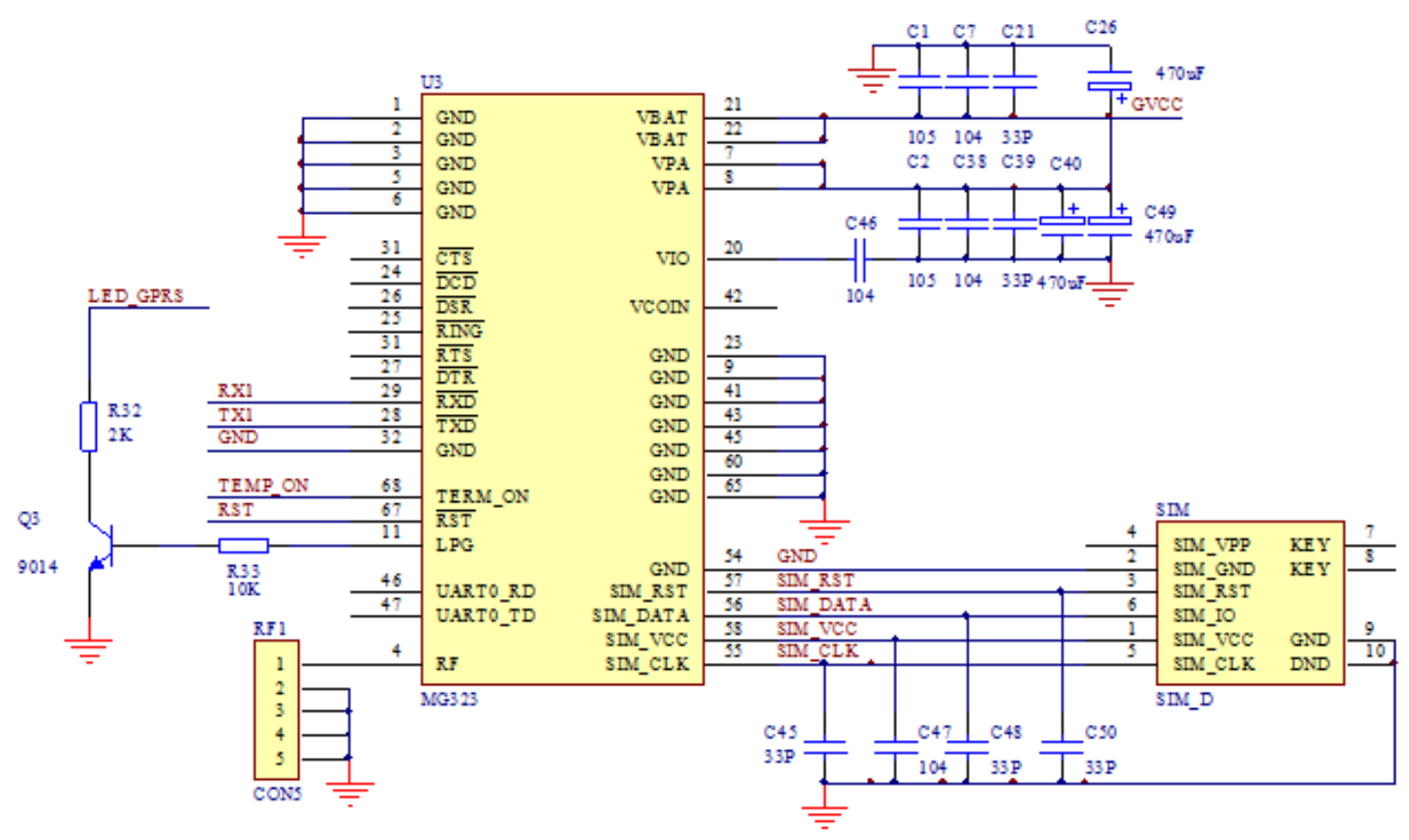

Figure 3: MG323 interface circuit principle diagram

\subsection{ZigBee Module Design}

SZ05 series embedded wireless communication module is divided into center coordinator, router and terminal node. These three types of equipment have different network functions. the Center coordinator is the central node of the network, responsible for the network launch organization, network maintenance and management functions; the router is responsible for the routing relay forwarding of the data, and the terminal node only sends and receives the data of this node [6]. Different device functions can be achieved through jumper settings or software configuration. SZ05-ZBEE Wireless Communication Module standard interface is normative, including power interface, data interface, control interface and system led interface and antenna interface, etc., The interface adopts standard 2.54 double row pins, and the system interface can be used socket or terminal block mode to the user system. The ZigBee module circuit schematic diagram is shown in Figure 4.

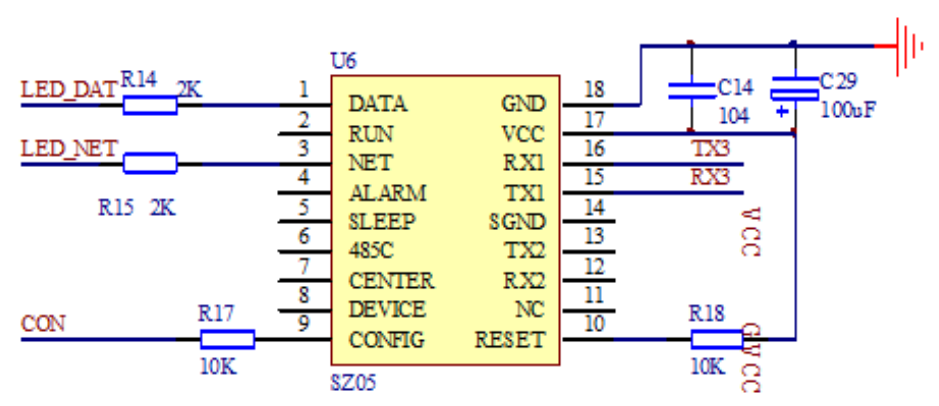

Figure 4: Zigbee module circuit principle diagram 


\section{SYSTEM SOFTWARE DESIGN}

\subsection{Overall system software design}

Building concentrator system software design mainly includes MG323GPRS module software design, Zigbee module software design and PC software design. The overall software design structure of the system is shown in Figure 5.

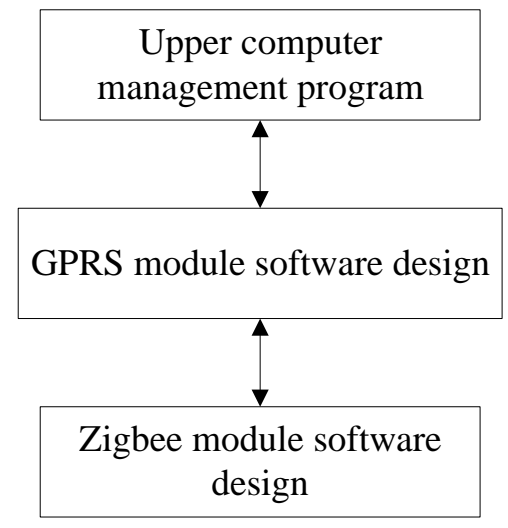

Figure 5: The overall system software

SZ05 series Zigbee modules are divided into the software design of the central coordinator, routers and terminal nodes. The software is designed according to the different functions of the module, and different types of programs are selected for downloading [7]. The main function of the SZ05Zigbee software is to detect the heat supply status and receive the control commands at the same time to realize the control of the valve opening degree. The GPRS module communicates with the ZigBee coordinator through the serial port. The main function of the GPRS module which plays a role of a transfer device, is to transfer the data collected by ZigBee, and then pass it to the host computer through the existing 3G/4G network. The main function of the upper computer system software is to design the human-computer interaction interface and realize data management and system control functions [8].

\subsection{Zigbee module software design}

The SZ05 series wireless embedded communication module adopts the standard Z-BEE wireless technology. The Zigbee network is established after the initialization of the coordinator. At the same time, the equipment is found to establish the communication link and read the serial data connected with the STM32. If the network data is used, the data frame is sent out, then the data of the RF buffer is read, and if the network data is used, the STM32 master chip is sent through the serial port.

\subsection{MG323GPRS module software design}

MG323GPRS module software design is a bridge for information exchange between the whole system. The GPRS module is placed on the scene. The main function is to transmit and process data collected by Zigbee coordinator and to command the Zigbee network. The GPRS module is placed in the system manager, through the serial port communication, to achieve the remote transmission of data and the release of information commands. The information transmission of the GPRS module is carried out through the AT instruction set. The concentrator uses the HUAWEI MG323 module, and the GPRS communication instruction is: ATD*99\# request GPRS service command, ATD*98\# request GPRS IP service command, AT+CRLP setting the wireless link protocol parameter command of non transmission call data service, and so on. Through the above analysis, the main function of the GPRS module is to connect Zigbee and management software to realize the remote control of data, so the communication protocol with high reliability and good security becomes very important. The working voltage of the MG323GPRS module is $3.3 \mathrm{~V}-$ $4.8 \mathrm{~V}$. It is recommended that the edge voltage should not be used [9]. The voltage fluctuation will cause unstable operation of the module, and the power supply recommends $3.8 \mathrm{~V} / 2 \mathrm{~A}$. The module opening process is shown in Figure 6. Adaptive baud rate, AT communication requires synchronous serial port baud rate process, which is shown in Figure 7.

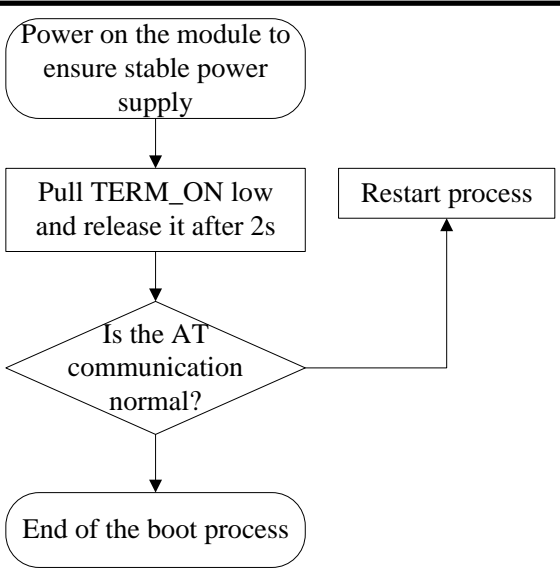

Figure 6: Module boot process

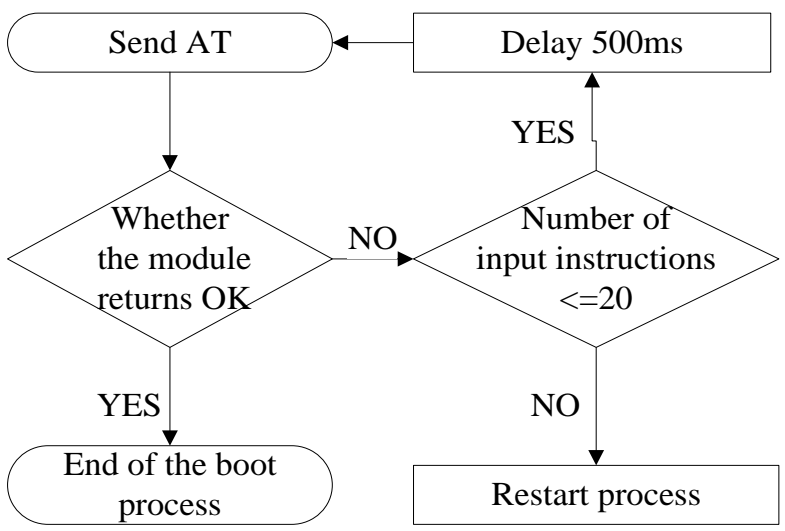

Figure 7: Synchronous serial port baud rate flow

\subsection{Computer software design}

The host computer programming is based on Web network service software platform. The working process of Web server is divided into 4 steps: the connection process, the request process, the response process and the closing connection [10]. It can support multiple processes, multiple threads and multiple processes combined with multiple threads. Information sharing can be achieved through simple programming. The GPRS module passes the information to the Web server through the 3G/4G network, then displays it on the display, and returns the regulation command back to the Zigbee module through the Web server.

\section{CONCLUSION}

This design of this research is based on the need of dynamically adjusting remote control heating. Through making use of Zigbee-based wireless sensor networks and GPRS wireless communication technology, an Internet - based system for heating and remote real-time monitoring has been constructed. On the one hand, the system can be used to manage and monitor users' hotlists effectively; on the other hand, it provides a basis for improving the performance of the central heating system. Because this system uses wireless data collection technology, the latest achievements of public wireless communication technology and micro-controllers, it not only has more advanced technology but also has a better practicality. Furthermore, it can save human force, material and financial resources required by remote monitoring of hot table data. A lot of valuable heat meter monitoring data can be acquired through using this system and it can provide evidences for improving the performance of the central heating system.

\section{REFERENCES}

[1] Huang, C.F., Lee, H.W., Tseng, Y.C. 2004. A two-tier heterogeneous mobile Ad Hoc network architecture and its load-balance routing problem. Mobile Networks and Applications, 9 (4), 379-391.

[2] Ruixiu, C. 2018. Zigbee and GPRS technology in hydrological monitoring system. Dandong hydrology bureau, Dandong, Liaoning 02, 118001.

[3] Yun, C. 2011. Research on remote real-time monitoring system of heat meters based on Internet of things. Yanshan University. 
[4] Ying, H., Anxing, Z., Shuai, Y., Yip, W. 2015. The campus street lamp control system based on Zigbee and GPRS. Shandong Jianzhu University.

[5] Leon, R.A., Vittal, V., Manimaran, G. 2007. Application of sensor Network for Secure Electric Energy Infrastructure. IEEE Tran. Power Delivery, 22 (2), 1021-1028.

[6] GSM 04.64, GPRS. 1998. Mobile Station-Serving GPRS Support Node (MS_SGSN) Logical Link Control (LLC) Layer Specification, 23-26.

[7] Yi, Z., Bo, Y.P., Quan, G. 2012. Design of street lamp control system based on Zig Bee network. Measurement and control technology and instrumentation, 38 (10), 77 - 80.

[8] Tiantian, W. 2007. Research on wireless heating network monitoring system based on Zigbee. Nanjing University of Science and Technology.

[9] Pei, H. 2018. Research on underground pipeline monitoring system based on Zigbee and GPRS. Journal of office automation.
[10] Haiyan, H. 2017. Design of data concentrator based on GPRS wireless module. Huizhou Zhongcheng Electronic Technology Co., Ltd, 08.

\section{ABOUT THE AUTHORS}

Xuezhong Ai (1970-): Male, Yushu City, Jilin Province, professor of Jilin Institute of Chemical Technology, mainly researching on industrial automatic control and machine vision.

Wei Liu (1979-), director of the school of information and control, jilin university of chemical technology, mainly researching on the direction of intelligent control devices.

Fusheng Fan, 2017 graduate student of jilin university of chemical technology, mainly researching on industrial automatic control and machine vision.

Gang Fang, 2015 undergraduate of jilin university of chemical technology. 